\title{
Early symptom non-improvement and aggravation are associated with the treatment response to SSRIs in MDD: a real-world study
}

This article was published in the following Dove Medical Press journal: Neuropsychiatric Disease and Treatment

\author{
Hsinsung Yuan ${ }^{1,2}$ \\ Xiao Zhu' \\ Qiang Luo ${ }^{3,4}$ \\ Alice Halim ${ }^{5}$ \\ Michael Halim ${ }^{5}$ \\ Hao Yao ${ }^{5}$ \\ Yiyun Cai ${ }^{1,6}$ \\ Shenxun Shi ${ }^{1,6}$
}

'Psychiatry Department of Huashan Hospital, Fudan University, Shanghai, People's Republic of China;

${ }^{2}$ Psychiatry Department of Nanjing Brain Hospital, Nanjing, People's Republic of China; ${ }^{3}$ Institute of Science and Technology for BrainInspired Intelligence, Fudan University, Shanghai, People's Republic of China; ${ }^{4}$ Key Laboratory of Computational Neuroscience and Brain-Inspired Intelligence (Ministry of Education), Fudan University, Shanghai, People's Republic of China; ${ }^{5}$ Shanghai Medical College, Fudan University, Shanghai, People's Republic of China; ${ }^{6}$ Shanghai Mental Health Center, Shanghai, People's Republic of China

\begin{abstract}
Purpose: Early improvement in major depressive disorder is defined as a reduction of $\geq 20 \%$ in the 17-item Hamilton Depression Rating Scale (HAM-D-17) score at the second week after initiation of treatment, predicting long-term treatment response. However, there remains no effective strategy for switching medications when a patient fails to reach early improvement at the second week. This study focused on the predictive value of early symptom changes in each item of the HAM-D-17 scale for treatment response to selective serotonin reuptake inhibitor (SSRI) monotherapy and to provide a reference for switching antidepressants to enhance early treatment efficacy.
\end{abstract}

Patients and methods: Our study was an observational, real-world study that enrolled 90 treatment-naïve patients experiencing their first episode of major depressive disorder in the outpatient department of Huashan Hospital. Patients who did not achieve the threshold of early improvement in the second week after starting treatment were switched to alternative SSRI monotherapy. Patient follow-up occurred at 2, 4, 8, and 12 weeks after the initiation of treatment. We analyzed the relationship between the change in each symptom on the HAM-D-17 scale and treatment efficacy.

Results: Early improvement predicted the treatment response at 12 weeks $\left(\chi^{2}=19.249\right.$, $P<0.001$ ), whereas early non-improvement in insomnia and anxiety was associated with a poor response $(\mathrm{OR}=9.487,95 \% \mathrm{CI}: 1.312-68.588$ and $\mathrm{OR}=12.947,95 \% \mathrm{CI}: 1.99-82.246$, respectively). At week 2, general somatic symptom aggravation was associated with a poorer response ( $\mathrm{OR}=73.337,95 \% \mathrm{CI}: 2.232->999.999)$; treatment-emergent headache and tremor were associated with treatment efficacy ( $\mathrm{t}=-9.521, P<0.001$ and $\mathrm{t}=3.660, P=0.001$, respectively). In addition, the increase in suicidal thoughts, once treatment began, had no relationship with the treatment response $(\mathrm{OR}=0.821, P=0.872)$.

Conclusion: This study suggested that patients with early non-improvement in insomnia and anxiety were not suitable for switches in SSRI monotherapy. Patients with treatment-emergent symptoms, especially headaches and tremors, were not suitable for switching from monotherapy to another SSRI.

Keywords: early improvement, major depressive disorder, antidepressants, selective serotonin reuptake inhibitors

\section{Plain language summary}

Psychiatry Department of Huashan Hospital, Fudan University, No 12 Urumqi Middle Road, Jingan District, Shanghai, People's Republic of China Tel +86 I38 I620 3 I7|

Email shishenxun@I63.com
There is still no effective strategy for switching antidepressant medications when a patient fails to reach early improvement after the second week of treatment. Our study suggested that a patient who fails to reach early improvement at the second week, with no improvement in insomnia and anxiety or with treatment-emergent headache and tremor, experienced a poorer 
treatment response as a result of switching the original selective serotonin reuptake inhibitors to an alternative.

\section{Introduction}

Selective serotonin reuptake inhibitors (SSRIs) are first-line pharmacotherapeutic agents for patients with moderate to severe major depressive disorder (MDD). ${ }^{1}$ However, symptom reduction is often less than ideal, with approximately half of all patients responding to the first intervention. ${ }^{2}$ The longer a patient fails to respond to an antidepressant, the greater the likelihood of nonresponse is. ${ }^{3}$ As a result, several studies have indicated that an early drug response, occurring within the first 2 weeks of antidepressant treatment, may predict treatment outcome. An improvement of $20 \%$ in the score on the 17-item Hamilton Depression Scale (HAM-D-17) at the second week of antidepressant treatment is a predictor of eventual response and remission. ${ }^{4-11}$

For patients who fail to achieve early improvement, there remains no proper treatment strategies to enhance treatment efficacy. Studies have shown that there is similar efficacy between most of the antidepressants and psychological counselling. In addition, there is no known effective way to improve the efficacy. ${ }^{12}$ Nevertheless, many researchers are still working toward improving the efficacy through research with regard to how efficacy is influenced by subclinical symptom manifestations, ${ }^{13,14}$ including personality, ${ }^{12}$ physical pain, ${ }^{12}$ obsessive symptoms, ${ }^{12}$ and subclinical manic syndrome, ${ }^{15,16}$ which could help us identify endophenotypes of MDD to improve treatment efficacy. ${ }^{17,18}$

Consistent early patterns of symptom improvement are factors to help us evaluate treatment efficacy. ${ }^{19-21}$ In addition, early symptom non-improvement and symptom aggravation are important factors that should be considered with regard to overall quality of life. ${ }^{22}$ These treatment-emergent symptoms were often attributed to adverse drug reactions and were evaluated with treatment tolerance. The treatment-emergent symptoms, which were associated with an inferior treatment response, may also be attributed to excessive worry about the disease, more emphasis on bodily concerns, and the course of disease progression, all of which consequently result in a lack of confidence toward medication. ${ }^{23}$

In the present study, we used an observational realworld study design, which better reflected the complexity of youth outpatients, as this age range is arguably at risk for bipolar disorder and often excluded from MDD cohorts. We evaluated early symptom changes in the HAM-D-17 and recorded personality traits, obsessive symptoms, social function, and adverse drug reactions simultaneously.
We examined symptoms that did not improve (nonimprovement) and symptoms that worsened (aggravation) to characterize early symptom changes. We hypothesized that, in addition to early improvement, early symptom aggravation was associated with efficacy, which contributed to predicting future outcomes. Our primary focus was to identify patients who were not properly treated with SSRIs based on early symptom non-improvement and early symptom aggravation.

\section{Patients and methods Participants}

The patients considered for inclusion were screened for this prospective, observational real-world study, which aimed to investigate early improvement. To meet the inclusion criteria, all enrolled patients were required to be within the age range of 18-55 years, be experiencing their first episode of MDD, be naïve to treatment, and have a baseline HAM$D-17$ score $\geq 18$. A trained psychiatric student conducted a structured interview using the Chinese version of the MiniInternational Neuropsychiatric Interview for all participants. A diagnosis was made according to the Diagnostic and Statistical Manual of Mental Disorders, 4th edition (DSM-IV) criteria by a trained, senior-level psychiatrist. Informed consent was obtained from all patients on their first visit. Exclusion criteria included 1) a history of psychoactive substance abuse; 2) current pregnancy or lactation; 3) any physical diseases assessed by personal history; and 4) a history of neurological disorders, other existing psychiatric disorders, or cardiovascular diseases.

This study was approved by the ethics boards of Huashan Hospital and Fudan University. All participants provided their written informed consent (Number: 2017-276). The authors assert that all procedures contributing to this work complied with the ethical standards of the relevant national and institutional committees on human experimentation and with the Declaration of Helsinki of 1975, as revised in 2008.

\section{Medication}

Patients received first-line monotherapy with a SSRI after diagnosis. The SSRIs used in this study included escitalopram, sertraline, and paroxetine, which reported similar effectiveness and tolerance in treating MDD. The treatment started with lowest commercially available unit dose and reached the effective dose on the third day of treatment. Between the 14th and 20th day of treatment, patients who failed to reach the criteria of early improvement were administered an alternative first-line SSRI medication. 


\section{Scale assessments}

During the study, the Eysenck Personality Questionnaire (EPQ), ${ }^{24}$ Yale-Brown Obsessive Compulsive Scale (Y-BOCS), ${ }^{25}$ Short Form of the Childhood Trauma Questionnaire (CTQ-SF), ${ }^{26}$ Patient Health Questionnaire-9 (PHQ-9), ${ }^{27}$ Generalized Anxiety Disorder Scale-7 (GAD-7), ${ }^{28}$ and Liebowitz Social Anxiety Scale (LSAS) ${ }^{29}$ were completed by all non-schizophrenic, literate patients in the Huashan Hospital Psychological Counseling Expert Outpatient department on Monday mornings and Thursday afternoons. Two trained psychiatric postgraduate students assessed the patients via the 17-item Hamilton Depression Scale (HAMD-17) and Hamilton Anxiety Rating Scale (HAM-A). ${ }^{30}$ Patients needed to provide marital status, educational level, occupation, family history, presence of somatic diseases, their own attribution of their symptoms (such as occupational pressure, divorce, and interpersonal relationships), correlations between symptoms and menstrual cycle, family history of mental disease, tobacco and alcohol use history, and so on. In addition, the patients' medical histories collected at the visit included the duration for which symptoms had been present and information about previous mental diseases. In this study, at the initial visit and at weeks 2 , 4,8 , and 12 of treatment, PHQ-9 and GAD-7 were used to assess patients' self-rated depression and anxiety symptoms, and the Asberg's Antidepressant Side-Effect Rating Scale (SERS) was used to assess adverse drug events since the previous visit.

\section{Statistical analysis}

In accordance with previous, similar work, early improvement was defined as a $\geq 20 \%$ reduction in the HAM-D-17 total score at week 2 , and a treatment response was defined as a 50\% reduction in the HAM-D-17 score at week 12 . Baseline sociodemographic and clinical characteristics were compared between the response group and non-response group by independent $t$-tests and the chi-squared test or Fisher's exact test for continuous and categorical variables, respectively. Sensitivity, specificity, positive predictive value (PPV), and negative predictive value (NPV) of each symptom for early improvement and early aggravation were calculated for predicting treatment response. Scores for each symptom were estimated by means of two data sets: 1) an observed case analysis, which included all participants without missing observations at each time point and 2) a last observation carried forward analysis, in which the score at the previous visit was adopted and used thereafter in the case of premature attrition. An independent $t$-test for continuous variables and a chi-squared test or Fisher's exact test for categorical variables were performed to compare differences between the two groups. Multiple logistic regression analyses were performed to evaluate the association between individual symptom improvement and aggravation in the early phase and response by the end of the treatment study period. The area under the receiver operating characteristic curve was used to confirm the predictive value. We used baseline sociodemographic, clinical characteristics, and adverse drug events at the second week for analysis to identify the influential factors of the symptom changes that were significantly related to treatment response, and we used a mediation analysis via SPSS PROCESS to analyze the structural relationships between the influential factors, symptom changes, and the treatment response. A two-sided $P$-value $<0.05$ was considered statistically significant. Statistical analyses were performed with IBM SPSS Statistics version 22.0.

\section{Results \\ Subject characteristics}

A total of 84 patients were enrolled in the study, with $69 \%$ women and an average age of 30.6 years. From May to November 2017, there were 126 first-episode MDD patients who visited Huashan Hospital affiliated with Fudan University. As shown in Table 1, 90 patients were willing to undergo laboratory tests and start antidepressant treatment. Of the 90 patients who were willing to start medication, six (7\%) were reluctant to return and were no longer contacted for follow-up. Of the 84 patients enrolled in follow-up, 56 (66\%) achieved early improvement during the second week of treatment, while 28 patients did not meet the early improvement criteria at the second week of treatment (Figure 1). Table 1 shows that the age, sex, baseline depression and anxiety scores, duration of illness, and social function deficits of the enrolled patients were all independent of whether there was an effective response at week 12 of treatment, with social avoidance at baseline and the psychoticism personality trait in the Eysenck Personality Test related to the treatment efficacy $(\mathrm{t}=2.534, P=0.013$ and $\mathrm{t}=3.115, P=0.003$, respectively).

\section{Early symptom improvement and treatment response}

Early improvement, that is, a 20\% reduction in the HAM-D-17 score at the second week of treatment, predicted treatment response at week $12\left(\chi^{2}=19.249, P<0.001\right)$ and had a PPV of $86 \%$ (Table 2). Since another SSRI was substituted in the second week, the NPV was only $33 \%$. The area under the 
Table I Clinical features between the response group and the non-response group at baseline

\begin{tabular}{|l|l|l|l|l|}
\hline & Response $(\mathbf{n}=\mathbf{5 9})$ & Non-response $(\mathbf{n}=\mathbf{2 5})$ & t or $\chi^{2}$ & $\boldsymbol{P}$-value \\
\hline Age (mean \pm SD, years) & $31.31 \pm 9.43$ & $27.12 \pm 9.01$ & -1.883 & 0.063 \\
\hline Sex (female/male) & $41 / 18$ & $18 / 7$ & 0.053 & 0.818 \\
\hline Course (mean \pm SD, months) & $10.75 \pm 16.71$ & $16.96 \pm 21.01$ & 1.311 & 0.198 \\
\hline HAMD-I7 & $24.15 \pm 5.05 I$ & $25.40 \pm 5.212$ & 1.025 & 0.308 \\
\hline HAMA & $22.07 \pm 6.349$ & $24.16 \pm 5.878$ & 1.411 & 0.162 \\
\hline GCI & $4.56 \pm 1.193$ & $4.96 \pm 1.098$ & 1.440 & 0.154 \\
\hline SDSS & $6.59 \pm 4.751$ & $7.44 \pm 4.976$ & 0.074 & 0.462 \\
\hline LSAS social anxiety & $21.14 \pm 14.56$ & $28.42 \pm 18.92$ & 1.659 & 0.106 \\
\hline Social avoidance & $20.67 \pm 15.04$ & $31.04 \pm 18.97$ & 2.534 & $0.013 *$ \\
\hline EPQ E & $7.00 \pm 3.593$ & $5.88 \pm 4.503$ & -1.205 & 0.232 \\
\hline P & $5.28 \pm 3.031$ & $7.64 \pm 3.487$ & 3.115 & $0.003 * *$ \\
\hline N & $16.64 \pm 5.405$ & $18.400 \pm 4.203$ & 1.450 & $0.15 I$ \\
\hline L & $11.59 \pm 3.603$ & $10.92 \pm 3.957$ & -0.722 & 0.474 \\
\hline
\end{tabular}

Notes: Response, a reduction in HAMD-17 $\geq 50 \%$. $* P<0.05$; **P $<0.0$ I.

Abbreviations: HAMD-17, I7-item Hamilton Depression Scale; HAMA, Hamilton Anxiety Scale; CGI, Clinical Overall Impression Scale; Y-BOCS, Yale-Brown Obsessivecompulsive Scale; LSAS, Liebowitz Social Anxiety Scale; SDSS, Social Function Defect Rating Scale; EPQ, Eysenck Personality Questionnaire; E, extroversion; P, psychoticism; $\mathrm{N}$, neuroticism; L, lie.

receiver operating characteristic curve for early improvement predicting a treatment response was 0.747 . Early improvement was not associated with age, sex, duration of disease, baseline depression score, baseline anxiety score, social function deficit, social anxiety, and so on. The results of the independent chi-squared test showed that sad mood, feelings of guilt, agitation, anxious mood, and somatic anxiety were more effectively changed in the group that showed early improvement than in the non-improvement group, and the differences were statistically significant $\left(\chi^{2}=9.275\right.$, $\chi^{2}=3.906, \chi^{2}=4.953, \chi^{2}=12.089, \chi^{2}=4.257, \chi^{2}=6.980$, respectively; $P<0.05$; Table 3 ). In multiple logistic regression analysis, improvement in difficulty with falling asleep (OR $=9.487,95 \%$ CI: 1.312-68.588) and mood anxiety (OR $=12.947,95 \%$ CI: 1.99-82.246) were selected as independent variables, using the minimum Akaike's information criterion by the stepwise method (Table 4; Figure 2). For these factors, the improvement in patients with the neuroticism personality trait was less pronounced $(\mathrm{t}=3.566$, $P=0.001$, 95\% CI: -5.139 to 1.453$)$. Mediation analysis

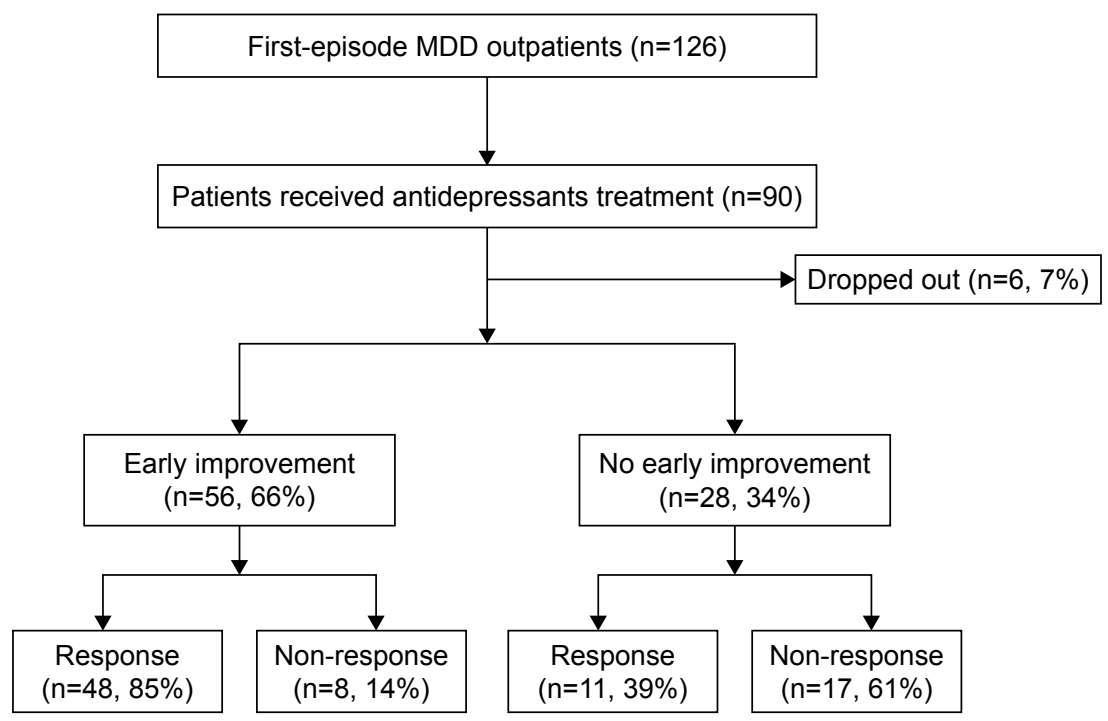

Figure I Study flowchart. There were 126 outpatients with MDD who visited Huashan Hospital during May to November 2017 . Among these patients, 90 of them received medication. These patients received monotherapy with SSRIs, and they were followed up at week 2, 4, 8, and I 2 of treatment. There were $66 \%$ patients who showed early improvement and $70 \%$ patients showed a response at week 12.

Abbreviations: MDD, major depressive disorder; SSRI, selective serotonin reuptake inhibitor. 
Table 2 Clinical features between early improvement or non-improvement

\begin{tabular}{|c|c|c|c|c|}
\hline & Early improvement $(n=56)$ & Non-improvement $(\mathrm{n}=\mathbf{2 8})$ & t or $\chi^{2}$ & $P$-value \\
\hline Age (mean $\pm S D$, years) & $30.57 \pm 9.63$ & $29.04 \pm 9.19$ & 0.699 & 0.486 \\
\hline Sex (female/male) & $38 / 18$ & $2 \mathrm{I} / 7$ & 0.456 & 0.616 \\
\hline Course (mean $\pm S D$, month) & $12.00 \pm 17.76$ & $13.4 \mid \pm 19.34$ & -0.287 & 0.775 \\
\hline HAMD-I7 & $24.13 \pm 5.15$ & $25.32 \pm 5.00$ & -1.014 & 0.314 \\
\hline HAMA & $22.11 \pm 6.10$ & $23.86 \pm 6.49$ & -1.213 & 0.229 \\
\hline $\mathrm{GCl}$ & $4.61 \pm 1.17$ & $4.82 \pm 1.18$ & -0.787 & 0.434 \\
\hline Y-BOCS & $9.55 \pm 7.90$ & $9.63 \pm 9.51$ & -0.035 & 0.972 \\
\hline SDSS & $6.57 \pm 4.9$ & $7.76 \pm 4.9$ & -0.642 & 0.523 \\
\hline LSAS social anxiety & $23.60 \pm 16.48$ & $23.40 \pm 16.43$ & 0.050 & 0.960 \\
\hline Social avoidance & $23.88 \pm 18.18$ & $24.48 \pm 15.08$ & -0.143 & 0.887 \\
\hline EPQ E & $7.05 \pm 3.70$ & $5.89 \pm 4.23$ & 1.290 & 0.201 \\
\hline $\mathrm{P}$ & $5.62 \pm 3.15$ & $6.71 \pm 3.62$ & -1.424 & 0.158 \\
\hline $\mathrm{N} * *$ & $16.18 \pm 5.63$ & $19.11 \pm 3.17$ & 3.025 & 0.003 \\
\hline L & $\mid 1.31 \pm 3.72$ & $11.54 \pm 3.72$ & -0.262 & 0.794 \\
\hline Response rate at week $12 * * *$ & $86 \%$ & $33 \%$ & 19.259 & $<0.001$ \\
\hline Remission rate at week $12 * * *$ & $63 \%$ & $11 \%$ & 25.11 & $<0.001$ \\
\hline
\end{tabular}

Notes: Response, a reduction in HAMD-17 $\geq 50 \%$. **P $<0.01$; ***P $<0.001$.

Abbreviations: Course, course of disease; HAMD-17, I7-item Hamilton Depression Scale; HAMA, Hamilton Anxiety Scale; CGI, Clinical Overall Impression Scale; Y-BOCS, Yale-Brown Obsessive-Compulsive Scale; LSAS, Liebowitz Social Anxiety Scale; SDSS, Social Function Defect Rating Scale; EPQ, Eysenck Personality Questionnaire;

$\mathrm{E}$, extroversion; $\mathrm{P}$, psychoticism; N, neuroticism; L, lie.

showed that no improvement in difficulty falling asleep was a mediation factor affecting the treatment response in patients with the neuroticism personality trait $\left(\mathrm{c}^{\prime}=-0.013\right.$, Bootstrap CI: -0.1090 to -0.0008$)$. The factors related to mood anxiety non-improvement were the psychoticism personality trait ( $\mathrm{t}=-2.937, P=0.004,95 \% \mathrm{CI}:-3.4721$ to 0.6689), physical fatigue after taking the drug $(\mathrm{t}=-2.708$, $P=0.010,95 \%$ CI: -0.9131 to 0.1334$)$, and total side effect score ( $\mathrm{t}=-3.075, P=0.004,95 \%$ CI: -6.3582 to 1.3154 ). However, mediation analysis showed that these related factors were not mediators of the relationship between symptom improvement and the response at week 12 of treatment.

\section{Early symptom aggravation and treatment response}

Independent chi-squared test did not demonstrate that any early symptom aggravation was related to the treatment response. However, in multiple logistic regression, early symptom aggravation in general somatic symptoms was related to the treatment response $(\mathrm{OR}=73.337,95 \% \mathrm{CI}$ : 2.232->999.999; Table 5; Figure 2). The factors associated with general somatic symptom aggravation were depression fluctuations and menstrual cycle ( $\mathrm{t}=4.350, P<0.001,95 \%$ CI: $0.148-0.401)$, headache at the second week $(\mathrm{t}=-9.521$, $P<0.001,95 \%$ CI: -1.546 to 1.004$)$ and tremor at the second week ( $\mathrm{t}=3.660, P=0.001,95 \%$ CI: $0.131-0.454)$. Mediation analysis showed that these related factors were not mediation factors affecting the treatment response at week 12 . In addition, six (7\%) patients were observed to have worsened suicidal ideation at the second week of treatment, but this study did not show that aggravation in suicidal ideation was associated with efficacy $\left(\chi^{2}=0.420, P=0.522\right)$.

\section{Discussion}

This study was the first of its kind to describe first-episode MDD in youth patients who had no early improvement after SSRI monotherapy and were switched to treatment with another SSRI. Our study tried focusing on early treatmentemergent symptoms of SSRI to discuss the influence of symptom improvement on treatment effect. We distinguished early symptom improvement from early symptom aggravation and described the early symptom improvement from antidepressant treatment in each category. We analyzed the clinical features that can predict treatment effect from various clinical features very elaborately and identified the patients whose treatment effect was less than favorable among those who had no early improvement in a practical way to provide reference for the improvement of the early treatment effect of first-episode MDD patients as soon as possible. These results can be generalized to youth with first-episode MDD with or without anxiety, including MDD patients with social anxiety, obsession, smoking, and panic attack. This research was done at the psychiatric outpatient departments of general hospitals, excluding inpatient wards, so the study results are not applicable to patients with psychotic symptoms, pregnant and postpartum patients, patients using psychoactive substances, inpatients, youth patients in their first episode, and elderly patients. 


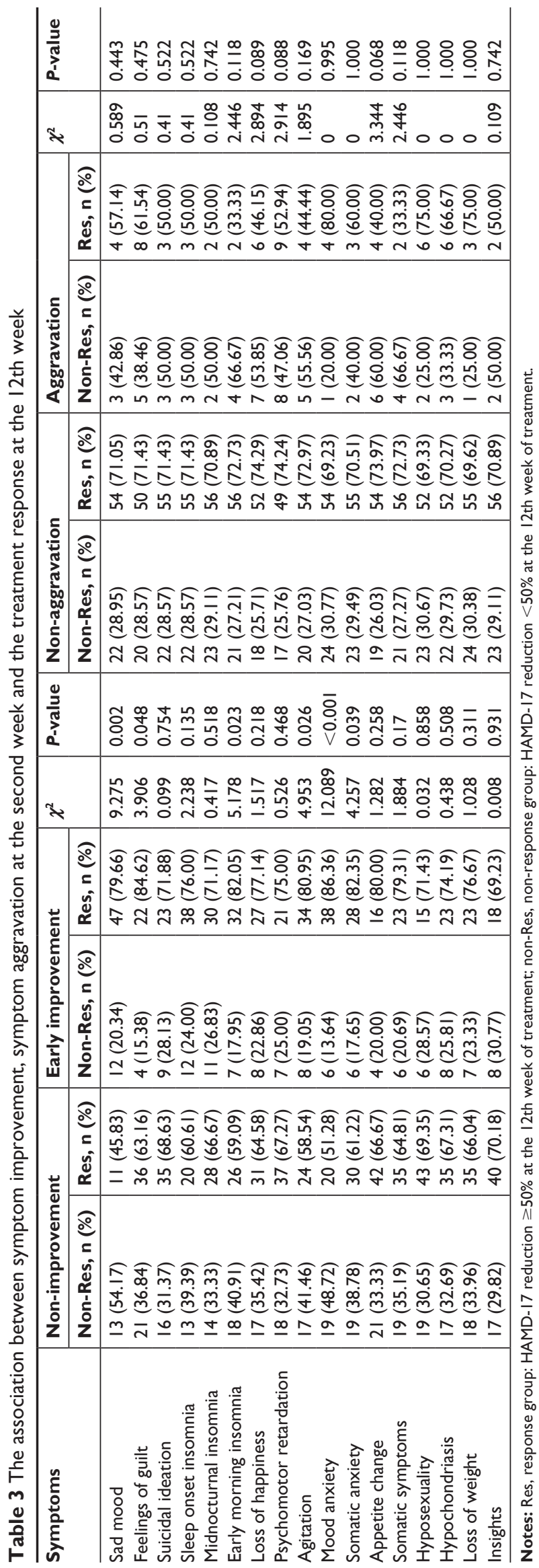

Table 4 A multiple logistic regression of the early symptom improvement in the 17 items of the Hamilton Depression Scale (HAMD-17) with subsequent treatment response

\begin{tabular}{l|l|l|l|l}
\hline Symptoms & \multirow{2}{*}{ OR } & \multicolumn{2}{|l|}{$\mathbf{9 5 \%}$ CI } & \multirow{2}{*}{ P-value } \\
\cline { 3 - 4 } & & Lower & Upper & \\
\hline Sad mood & 3.568 & 0.712 & 17.866 & 0.122 \\
Feelings of guilt & 1.402 & 0.249 & 7.899 & 0.702 \\
Suicidal ideation & 1.366 & 0.312 & 5.982 & 0.679 \\
Sleep onset insomnia & 9.487 & 1.312 & 68.588 & $0.026^{*}$ \\
Midnocturnal insomnia & 0.594 & 0.106 & 3.343 & 0.555 \\
Early morning insomnia & 4.474 & 0.702 & 28.525 & 0.113 \\
Loss of happiness & 0.759 & 0.164 & 3.508 & 0.724 \\
Psychomotor retardation & 0.957 & 0.22 & 4.163 & 0.954 \\
Agitation & 2.787 & 0.516 & 15.04 & 0.233 \\
Mood anxiety & 12.947 & 1.99 & 84.246 & $0.007 * *$ \\
Somatic anxiety & 2.512 & 0.472 & 13.358 & 0.280 \\
Appetite change & 0.738 & 0.115 & 4.724 & 0.749 \\
Somatic symptoms & 0.828 & 0.155 & 4.426 & 0.825 \\
Hyposexuality & 1.548 & 0.275 & 8.714 & 0.620 \\
Hypochondriasis & $I .468$ & 0.251 & 8.584 & 0.670 \\
Loss of weight & 1.759 & 0.401 & 7.704 & 0.454 \\
Insights & 1.542 & 0.356 & 6.671 & 0.563 \\
\hline Notes: & & &
\end{tabular}

Notes: $* P<0.05 ; * * P<0.01$.

\section{Non-improvement in mood anxiety and difficulty falling asleep}

Mood anxiety ${ }^{31}$ and difficulty falling asleep ${ }^{32}$ were reported as symptoms that improved quickly ${ }^{33}$ and were associated with treatment response..$^{34,35}$ Early studies suggested that mood anxiety is one of the most common symptoms in patients with MDD, and early mood anxiety improvement plays an important role in early SSRI treatment efficacy. ${ }^{34}$ Most of the SSRIs seemed to have poor efficacy in treating anxiety, ${ }^{36}$ including aroused anxiety, which, especially in teenagers, was associated with treatment-emergent suicidal ideation. ${ }^{23,37,38}$

In our study, non-improvement in mood anxiety and non-improvement in difficulty falling asleep occurred independently, with $44 \%$ of patients showing non-improvement in mood anxiety, $27 \%$ of patients having difficulty falling asleep, and only $11 \%$ of patients showing both symptoms in early non-improvement. In our study, changes in these symptoms showed a feature independently associated with personality traits. Our study suggested that patients with poorer improvement in insomnia were associated with neuroticism personality traits, which were associated with slower improvement, ${ }^{39}$ and those with poorer improvement in mood anxiety had psychoticism personality traits. For patients who possess a psychoticism personality trait, which implies subclinical bipolarity, ${ }^{40}$ it may not be appropriate to use monotherapy with SSRIs if they fail to achieve early improvements in anxiety. 
Early symptoms non-improvement

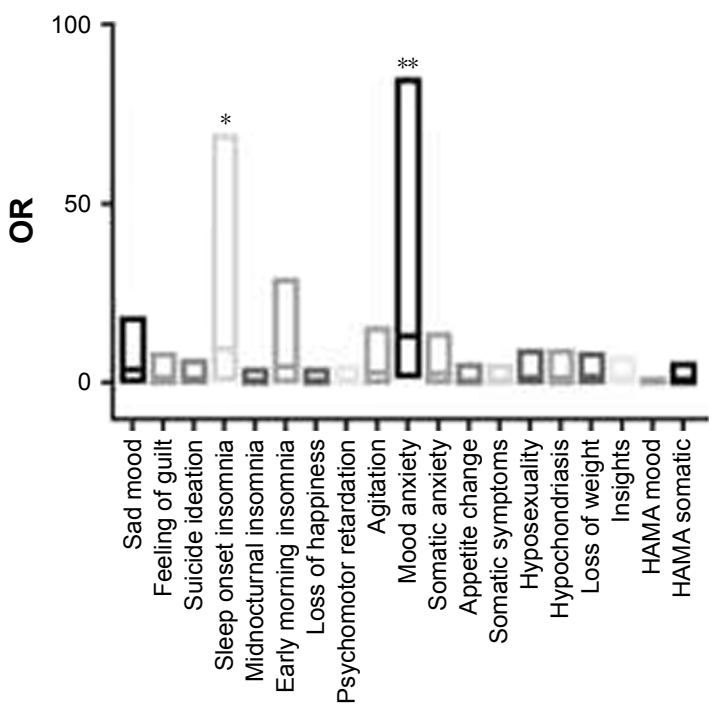

Early symptoms aggravation

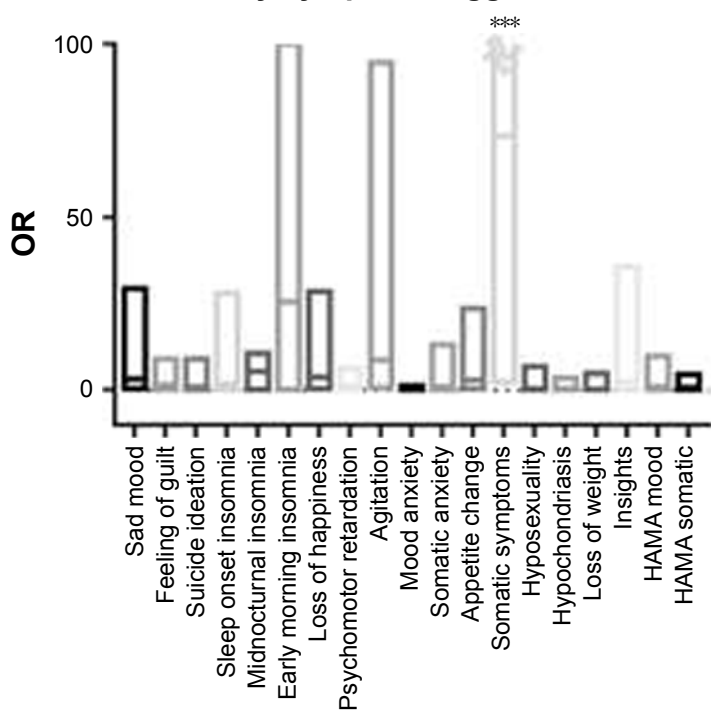

Figure 2 ORs for each early symptom improvement in the 17 items of the Hamilton Depression Scale (HAMD-I7) at week 2 to predict treatment response at week 12. Notes: $* P<0.05$; $* * P<0.01$; $* * * P<0.001$.

Abbreviation: HAMA, Hamilton Anxiety Scale.

\section{Early symptom aggravation in somatic symptoms}

In our study, general somatic symptom aggravation was related to treatment response. However, non-improvement in somatic symptoms had no relationship with treatment response. Treatment-emergent symptoms have troubled physicians for a long time. First, patients' adverse drug reactions are the most intuitive, but in many cases, it is not always possible

Table 5 A multiple logistic regression of the early symptom aggravation in the 17 items of the Hamilton Depression Scale (HAMD-17) with subsequent treatment response

\begin{tabular}{l|l|l|l|l}
\hline Symptoms & \multirow{2}{*}{ OR } & \multicolumn{2}{l|}{$\mathbf{9 5 \%} \mathbf{C l}$} & \multirow{2}{*}{ P-value } \\
\cline { 3 - 4 } & & Lower & Upper & \\
\hline Sad mood & 3.035 & 0.316 & 29.19 & 0.336 \\
Feelings of guilt & 1.53 & 0.263 & 8.917 & 0.636 \\
Suicidal ideation & 0.821 & 0.075 & 9.021 & 0.872 \\
Sleep onset insomnia & 1.813 & 0.118 & 27.873 & 0.670 \\
Midnocturnal insomnia & 0.017 & $<0.001$ & 10.497 & 0.214 \\
Early morning insomnia & 25.457 & 0.262 & $>999.999$ & 0.166 \\
Loss of happiness & 3.588 & 0.452 & 28.508 & 0.227 \\
Psychomotor retardation & 1.283 & 0.269 & 6.114 & 0.754 \\
Agitation & 8.639 & 0.79 & 94.425 & 0.077 \\
Mood anxiety & 0.042 & 0.001 & 1.213 & 0.065 \\
Somatic anxiety & 0.856 & 0.056 & 13.087 & 0.911 \\
Appetite change & 2.88 & 0.353 & 23.526 & 0.324 \\
Somatic symptoms & 73.337 & 2.232 & $>999.999$ & $0.016 *$ \\
Hyposexuality & 0.308 & 0.014 & 6.77 & 0.455 \\
Hypochondriasis & 0.31 & 0.028 & 3.373 & 0.336 \\
Loss of weight & 0.177 & 0.006 & 4.866 & 0.306 \\
Insights & 2.326 & 0.151 & 35.737 & 0.545 \\
\hline
\end{tabular}

Note: $* P<0.05$. to explain the adverse drug reactions alone. Second, MDD patients have diverse symptoms, including fatigue, headache, and dizziness which are often seen among adverse reactions to SSRI drugs and are also coincidentally symptoms of MDD. Patients may have symptom fluctuations after treatment. For example, menstrual cycle or temperature change may give patients the impression that the treatment aggravates the diseases. Furthermore, patients who are overly concerned about the treatment might end up experiencing suggestive adverse effects after learning about the drugs prescribed to them postdiagnosis either based on the patient information sheet that comes with those medications or based on information about those medications they researched on the Internet. In any case, if both early symptoms change and medical treatments occur simultaneously, it may cause patients to develop a biased impression toward the physician and the treatment prescribed.

Treatment-emergent somatic symptoms seemed to be related to poor response. ${ }^{41}$ The general somatic symptom aggravation was related to the adverse effects of headache and tremor at the second week of treatment. We also found that patients whose symptom fluctuations were related to the menstrual cycle tended to exhibit treatment-emergent somatic symptom aggravation. Headache is listed as a common side effect of most antidepressants as well. ${ }^{42}$ Some studies have reported that somatic symptom improvement in MDD often implies treatment response. ${ }^{43,44}$ It is often difficult to differentiate treatment-emergent somatic symptoms from adverse effects of antidepressants. The antidepressants 
that commonly cause headache are SSRIs and serotonin noradrenaline reuptake inhibitors, especially bupropion and escitalopram. ${ }^{45}$ A point worth mentioning is premenstrual syndrome/premenstrual dysphoric disorder is often regarded as an implication of bipolar disorder. ${ }^{46}$ Especially when SSRI treatment is ineffective and there are more adverse reactions after treatment, there is a high possibility that the patient may be suffering from bipolar depression and must be counselled.

\section{Limitations}

This study had several limitations. It was a single-center study. The characteristics of residents in general hospitals and the addresses of the hospitals in the city indicated that the patients had a higher level of education and social status. The general complaints of patients in general hospital psychiatric outpatient clinics are more pronounced. Many patients arrive after they have not been diagnosed with physical diseases in other departments. Therefore, the clinical features of most patients diagnosed with MDD were somatic pain and irritability. Symptoms such as psychomotor retardation and agitation are less obvious. This study was an observational study, and there was no control group with a delayed or modified switching time. This study was an observational study using drugs including paroxetine, escitalopram, and sertraline, and there was no difference in the effect of these drugs in the treatment of depression in a previous study.

\section{Conclusion}

In conclusion, early improvement can predict the 12 -week response of first-episode youth MDD patients to SSRI monotherapy. However, based on the medication change that occurred during the second week in this study, the negative predictive value of early improvement was significantly lower than that observed in other studies. It would not be a good choice to change medications to another SSRI for patients who had not reached early improvement in anxiety and difficulty falling asleep at week 2 and who had aggravation in headaches and tremors. In addition, the non-improvement of anxiety and insomnia was independent; anxiety was related to the psychoticism personality trait, and difficulty falling asleep was related to the neuroticism personality trait. Within treatment-emergent symptoms, headaches and tremors were related to a lower response rate, especially for patients whose depression was related to their menstrual cycle.

\section{Acknowledgments}

The authors are grateful to all participants for their cooperation in our study. The authors also gratefully acknowledge the support from the Institute of Science and Technology for Brain-Inspired Intelligence, Fudan University, Shanghai, China. Dr Luo was supported by Shanghai Municipal Science and Technology Major Project (No 2018SHZDZX01), National Key Research and Development Program of China (2018YFC0910500), the National Natural Science Foundation of China (grants 81873909 and 91630314), the Natural Science Foundation of Shanghai (grant 17ZR1444400), and the key project of Shanghai Science and Technology Innovation Plan (grant 16JC1420402).

\section{Author contributions}

H Yuan and S Shi designed the research; S Shi, X Zhu, Y Cai, and $\mathrm{H}$ Yuan performed outpatient care and recruited participants; H Yuan and Q Luo conducted the statistical analysis; H Yuan wrote the paper; S Shi and Q Luo edited the paper; A Halim, M Halim, and H Yao edited the English writing. All authors contributed to data analysis, drafting and revising the article, gave final approval of the version to be published, and agree to be accountable for all aspects of the work.

\section{Disclosure}

The authors report no conflicts of interest in this work.

\section{References}

1. Kennedy SH, Lam RW, McIntyre RS, et al. Canadian network for mood and anxiety treatments (CANMAT) 2016 clinical guidelines for the management of adults with major depressive disorder: section 3 . Pharmacological treatments. Can J Psychiatry. 2016;61(9):540-560. doi: $10.1177 / 0706743716659417$

2. Trivedi MH, Rush AJ, Wisniewski SR, et al. Evaluation of outcomes with citalopram for depression using measurement-based care in STAR*D: implications for clinical practice. Am J Psychiatry. 2006; 163(1):28-40. doi:10.1176/appi.ajp.163.12.2189

3. Nierenberg AA. Predictors of response to antidepressants general principles and clinical implications. Psychiatr Clin North Am. 2003; 26(2):345-352. doi:10.1016/S0193-953X(02)00105-3

4. Lam RW. Onset, time course and trajectories of improvement with antidepressants. Eur Neuropsychopharmacol. 2012;22(Suppl 3): S492-S498. doi:10.1016/j.euroneuro.2012.07.005

5. Stassen HH, Angst J, Hell D, Scharfetter C, Szegedi A. Is there a common resilience mechanism underlying antidepressant drug response? Evidence from 2848 patients. J Clin Psychiatry. 2007;68(8):1195-1205. doi:10.4088/JCP.v68n0805

6. Stassen HH, Delini-Stula A, Angst J. Time course of improvement under antidepressant treatment: a survival-analytical approach. Eur Neuropsychopharmacol. 1993;3(2):127-135. doi:10.1016/0924977X(93)90264-M

7. Szegedi A, Jansen WT, van Willigenburg AP, van der Meulen E, Stassen HH, Thase ME. Early improvement in the first 2 weeks as a predictor of treatment outcome in patients with major depressive disorder: a meta-analysis including 6562 patients. J Clin Psychiatry. 2009; 70(3):344-353. doi:10.4088/JCP.08m04839

8. Szegedi A, Muller MJ, Anghelescu I, Klawe C, Kohnen R, Benkert O. Early improvement under mirtazapine and paroxetine predicts later stable response and remission with high sensitivity in patients with major depression. J Clin Psychiatry. 2003;64(4):413-420. doi:10.4088/JCP.v64n0410

9. Tadic A, Helmreich I, Mergl R, et al. Early improvement is a predictor of treatment outcome in patients with mild major, minor or subsyndromal depression. J Affect Disord. 2010;120(1-3):86-93. doi:10.1016/j.jad. 2009.04.014 
10. Vermeiden M, Kamperman AM, Vulink ME, van den Broek WW, Birkenhager TK. Early improvement as a predictor of eventual antidepressant treatment response in severely depressed inpatients. Psychopharmacology. 2015;232(8):1347-1356. doi:10.1007/s00213014-3765-1

11. Kudlow PA, Cha DS, McIntyre RS. Predicting treatment response in major depressive disorder: the impact of early symptomatic improvement. Can J Psychiatry. 2012;57(12):782-788. doi:10.1177/ 070674371205701211

12. Eaton NR, Rodriguez-Seijas C, Carragher N, Krueger RF. Transdiagnostic factors of psychopathology and substance use disorders: a review. Soc Psychiatry Psychiatr Epidemiol. 2015;50(2):171-182. doi:10.1007/ s00127-014-1001-2

13. Krueger RF, Eaton NR. Transdiagnostic factors of mental disorders. World Psychiatry. 2015;14(1):27-29. doi:10.1002/wps.20175

14. Pezzoli P, Antfolk J, Santtila P. Phenotypic factor analysis of psychopathology reveals a new body-related transdiagnostic factor. PLoS One. 2017;12(5):e0177674. doi:10.1371/journal.pone.0177674

15. Angst J, Cui L, Swendsen J, et al. Major depressive disorder with subthreshold bipolarity in the National Comorbidity Survey Replication. Am J Psychiatry. 2010;167(10):1194-1201. doi:10.1176/appi.ajp. 2010.09071011

16. Angst J, Merikangas KR, Cui L, van Meter A, Ajdacic-Gross V, Rossler W. Bipolar spectrum in major depressive disorders. Eur Arch Psychiatry Clin Neurosci. 2018;268(8):741-748. doi:10.1007/s00406-018-0927-x

17. Hasler G, Drevets WC, Manji HK, Charney DS. Discovering endophenotypes for major depression. Neuropsychopharmacology. 2004; 29(10):1765-1781. doi:10.1038/sj.npp.1300506

18. Fernandes BS, Williams LM, Steiner J, Leboyer M, Carvalho AF, Berk M. The new field of 'precision psychiatry'. BMC Med. 2017;15(1):80 doi:10.1186/s12916-017-0849-x

19. Allen TA, Lam RW, Milev R, et al. Early change in reward and punishment sensitivity as a predictor of response to antidepressant treatment for major depressive disorder: a CAN-BIND-1 report. Psychol Med 2018:1-10. doi:10.1017/S003329171800244.

20. Nelson JC, Portera L, Leon AC. Are there differences in the symptoms that respond to a selective serotonin or norepinephrine reuptake inhibitor? Biol Psychiatry. 2005;57(12):1535-1542. doi:10.1016/j.biopsych. 2004.12.018

21. Katz MM, Tekell JL, Bowden CL, et al. Onset and early behavioral effects of pharmacologically different antidepressants and placebo in depression. Neuropsychopharmacology. 2004;29(3):566-579. doi:10.1038/sj.npp.1300341

22. Yang WC, Lin CH, Wang FC, Lu MJ. Factors related to the improvement in quality of life for depressed inpatients treated with fluoxetine. BMC Psychiatry. 2017;17(1):309. doi:10.1186/s12888-017-1489-6

23. Olfson M, Marcus SC, Shaffer D. Antidepressant drug therapy and suicide in severely depressed children and adults: a case-control study. Arch Gen Psychiatry. 2006;63(8):865-872. doi:10.1001/archpsyc.63.8.865

24. Eysenck H, Eysenck S. Manual of the Eysenck personality questionnaire. J Card Fail. 1975;20(5):S67.

25. Goodman WK, Price LH, Rasmussen SA, et al. The Yale-brown obsessive compulsive scale. I. Development, use, and reliability. Arch Gen Psychiatry. 1989;46(11):1006-1011. doi:10.1001/archpsyc.1989. 01810110048007

26. Bernstein DP, Stein JA, Newcomb MD, et al. Development and validation of a brief screening version of the childhood trauma questionnaire. Child Abuse Negl. 2003;27(2):169-190. doi:10.1016/S01452134(02)00541-0

27. Kroenke K, Spitzer RL, Williams JB. The PHQ-9: validity of a brief depression severity measure. J Gen Intern Med. 2001;16(9):606-613. doi:10.1046/j.1525-1497.2001.016009606.x

28. Spitzer RL, Kroenke K, Williams JB, Lowe B. A brief measure for assessing generalized anxiety disorder: the GAD-7. Arch Intern Med. 2006;166(10):1092-1097. doi:10.1001/archinte.166.17.1863
29. Beidel DC, Borden JW, Turner SM, Jacob RG. The social phobia and anxiety inventory: concurrent validity with a clinic sample. Behav Res Ther. 1989;27(5):573-576.

30. Maier W, Buller R, Philipp M, Heuser I. The Hamilton Anxiety Scale: reliability, validity and sensitivity to change in anxiety and depressive disorders. J Affect Disord. 1988;14(1):61-68.

31. Katz MM, Meyers AL, Prakash A, Gaynor PJ, Houston JP. Early symptom change prediction of remission in depression treatment. Psychopharmacol Bull. 2009;42(1):94-107.

32. Sakurai H, Uchida H, Abe T, et al. Trajectories of individual symptoms in remitters versus non-remitters with depression. J Affect Disord. 2013;151(2):506-513. doi:10.1016/j.jad.2013.08.002

33. Yu YM, Gao KR, Yu H, Shen YF, Li HF. Efficacy and safety of agomelatine vs paroxetine hydrochloride in Chinese Han patients with major depressive disorder: a multicentre, double-blind, noninferiority, randomized controlled trial. J Clin Psychopharmacol. 2018;38(3): 226-233. doi:10.1097/JCP.0000000000000878

34. Li G, Shen Y, Luo J, Li H. Efficacy of escitalopram monotherapy in the treatment of major depressive disorder: a pooled analysis of 4 Chinese clinical trials. Medicine (Baltimore). 2017;96(39):e8142. doi:10.1097/MD.0000000000008142

35. Zisook S, Lesser I, Stewart JW, et al. Effect of age at onset on the course of major depressive disorder. Am J Psychiatry. 2007;164(10): 1539-1546. doi:10.1176/ajp.2007.164.5.712

36. Bandelow B, Andersen HF, Dolberg OT. Escitalopram in the treatment of anxiety symptoms associated with depression. Depress Anxiety. 2007;24(1):53-61. doi:10.1002/(ISSN)1520-6394

37. Bottelier MA, Schrantee A, Van Wingen G, Ruhé HG, De Ruiter MB, Reneman L. A power analysis for future clinical trials on the potential adverse effects of SSRIs on amygdala reactivity. Front Biol. 2016; 11(3):256-259. doi:10.1007/s11515-016-1402-6

38. Ansorge MS, Zhou M, Lira A, Hen R, Gingrich JA. Early-life blockade of the 5-HT transporter alters emotional behavior in adult mice. Science. 2004;306(5697):879-881. doi:10.1126/science.306.5698.956a

39. Roberts BW, Luo J, Briley DA, Chow PI, Su R, Hill PL. A systematic review of personality trait change through intervention. Psychol Bull. 2017;143(2):117-141. doi:10.1037/bul0000088

40. Angst J, Gamma A, Benazzi F, Ajdacic V, Eich D, Rossler W. Toward a re-definition of subthreshold bipolarity: epidemiology and proposed criteria for bipolar-II, minor bipolar disorders and hypomania. J Affect Disord. 2003;73(1-2):133-146. doi:10.1016/S01650327(02)00322-1

41. Si T, Wang G, Yang F, et al. Efficacy and safety of escitalopram in treatment of severe depression in Chinese population. Metab Brain Dis. 2017;32(3):891-901. doi:10.1007/s11011-017-9992-5

42. Noseda R, Borsook D, Burstein R. Neuropeptides and neurotransmitters that modulate thalamo-cortical pathways relevant to migraine headache. Headache. 2017;57(Suppl 2):97-111. doi:10.1111/head.13083

43. Fava M, Mallinckrodt CH, Detke MJ, Watkin JG, Wohlreich MM. The effect of duloxetine on painful physical symptoms in depressed patients: do improvements in these symptoms result in higher remission rates? J Clin Psychiatry. 2004;65(4):521-530.

44. Lin $\mathrm{CH}$, Yen YC, Chen MC, Chen CC. Relief of depression and pain improves daily functioning and quality of life in patients with major depressive disorder. Prog Neuropsychopharmacol Biol Psychiatry. 2013;47:93-98. doi:10.1016/j.pnpbp.2013.08.003

45. Telang S, Walton C, Olten B, Bloch MH. Meta-analysis: second generation antidepressants and headache. J Affect Disord. 2018;236: 60-68. doi:10.1016/j.jad.2018.04.047

46. Kim DR, Czarkowski KA, Epperson CN, et al. The relationship between bipolar disorder, seasonality, and premenstrual symptoms. Curr Psychiatry Rep. 2011;13(6):500-503. doi:10.1007/s11920-011-0233-z 


\section{Publish your work in this journal}

Neuropsychiatric Disease and Treatment is an international, peerreviewed journal of clinical therapeutics and pharmacology focusing on concise rapid reporting of clinical or pre-clinical studies on a range of neuropsychiatric and neurological disorders. This journal is indexed on PubMed Central, the 'PsycINFO' database and CAS,

and is the official journal of The International Neuropsychiatric Association (INA). The manuscript management system is completely online and includes a very quick and fair peer-review system, which is all easy to use. Visit http://www.dovepress.com/testimonials.php to read real quotes from published authors.

Submit your manuscript here: http://www.dovepress.com/neuropsychiatric-disease-and-treatment-journal 